\title{
Desenvolvimento de salgadinhos extrusados a partir de fragmentos de arroz e de feijão
}

\section{Development of extruded snacks from rice and bean fragments}

\author{
Manoel Soares Soares Júnior ${ }^{1 *}$; Talita Pereira Baêta Santos²; Grazyella Fernandes \\ Pereira $^{3}$; Cibele da Silva Minafra ${ }^{4}$; Márcio Caliari ${ }^{5}$; Flávio Alves da Silva ${ }^{6}$
}

\section{Resumo}

Neste trabalho foram analisadas características físicas, sensoriais e nutricionais de salgadinhos extrusados obtidos a partir de diferentes formulações de farinhas de quirera de arroz (FQA) e de bandinha de feijão (FBF), visando ampliar a utilização destes subprodutos na alimentação humana. Para formulação dos salgadinhos utilizou-se delineamento inteiramente casualisado (DIC), com cinco tratamentos (0\% FQA/ $100 \%$ FBF; $25 \%$ FQA $/ 75 \%$ FBF; 50\% FQA/ 50\% FBF; 75\% FQA/ 25\% FBF e $100 \%$ FQA $0 \%$ FBF) e três repetições. Foram feitas as avaliações do índice de expansão (dezesseis replicatas), da composição centesimal e do valor energético (triplicatas). $O$ índice de expansão variou entre 2,0 e 3,9, e o tratamento $75 \%$ FQA/ 25\% FBF apresentou o maior valor. Com relação aos atributos sensoriais, o tratamento $100 \%$ FQA/ 0\% FBF apresentou melhor escore para os atributos aparência, aroma, textura e sabor, no entanto, este não apresentou diferença significativa em relação aos tratamentos $75 \%$ FQA/ $25 \%$ FBF e 50\% FQA/ 50\% FBF, com exceção do aroma. Com relação à composição centesimal, observou-se que houve um aumento de umidade e carboidratos e diminuição de cinzas, proteínas e extrato etéreo dos salgadinhos com a elevação da quantidade de FQA e redução da quantidade de FBF na formulação dos mesmos. Portanto, verificou-se que é viável o aproveitamento dos subprodutos de arroz e feijão como matéria-prima para desenvolver salgadinhos extrusados de alto valor nutricional e baixo custo, independente da proporção utilizada na formulação de farinhas de quirera de arroz e bandinha de feijão. Palavras- chave: Oryza sativa L, Phaseolus vulgaris L, subprodutos, extrusão

\begin{abstract}
\footnotetext{
1 Prof. e Pesquisador da Universidade Federal de Goiás, UFG. E-mail: mssoaresjr@hotmail.com

2 Aluna da Universidade Federal de Goiás, UFG. E-mail: talitapbs@hotmail.com

3 Aluna da Universidade Federal de Goiás, UFG. E-mail: grazyella1@hotmail.com

4 Pesquisadora da Universidade Federal de Goiás, UFG. E-mail: cibeleminafra@hotmail.com

5 Prof. e Pesquisador da Universidade Federal de Goiás, UFG. E-mail: macaliari@ig.com.br

6 Prof. e Pesquisador da Universidade Federal de Goiás, UFG. E-mail: flaviocamp@gmail.com

Autor para correspondência
}

In this work physical, sensorial and nutritional characteristics of extruded snacks, obtained from different formulations with flour of broken rice (BRF) and broken bean (BBF) were analysed aiming at extending the use of these byproducts in human feeding. For snacks formulation, completely randomized design (CRD), with five treatments (0\% BRF/ 100\% BBF; 25\% BRF/ 75\% BBF; 50\% BRF/ 50\% BBF; 75\% $\mathrm{BRF} / 25 \% \mathrm{BBF}$ and $100 \% \mathrm{BRF} / 0 \% \mathrm{BBF}$ ) and three repetitions, was used. Expansion index (sixteen replicates), proximate composition and energy value (triplicates) evaluations were made. Expansion index varied between 2,0 and 3,9, and the treatment $75 \% \mathrm{BRF} / 25 \% \mathrm{BBF}$ presented the biggest value. As for sensorial attributes, the treatment $100 \% \mathrm{BRF} / 0 \% \mathrm{BBF}$ presented the best score for appearance, aroma, texture and flavor attributes, however, no significant difference in relation to treatments $75 \%$ $\mathrm{BRF} / 25 \% \mathrm{BBF}$ and $50 \% \mathrm{BRF} / 50 \% \mathrm{BBF}$ with exception to aroma was presented. Regarding to proximate composition, it was observed that it had an increase of moisture and carbohydrates, and reduction of 
ashes, proteins and ether extract of the snacks with the increase of the amount of BRA and reduction of the amount of BBF in the formulation of the same ones. Therefore, it was verified that the use of byproducts of rice and beans is viable as raw material to develop extruded snacks of high nutritional value and low cost, independent of the amount used of broken rice and broken bean flour in the formulation.

Key words: Oryza sativa L, Phaseolus vulgaris L, byproduct, extrusion

\section{Introdução}

$\mathrm{O}$ arroz e o feijão destacam-se como importantes fontes de carboidratos e de proteínas na dieta alimentar do povo brasileiro e contribuem com cerca da metade da ingestão energética e protéica dos indivíduos. O Brasil é o maior produtor de arroz da América Latina e o nono maior produtor mundial (FAO, 2004), também é o segundo maior produtor de feijão do mundo, perdendo apenas para a Índia (SILVA; DEL PELOSO, 2006). Um aspecto importante, do ponto de vista nutricional, é o aumento da qualidade protéica de dietas mistas, contendo feijão e arroz, em decorrência do efeito complementar do alto conteúdo de lisina do feijão com o de aminoácidos sulfurados do arroz (LAJOLO; GENOVESE; MENEZES, 1996).

Como o arroz e o feijão comercializados no país passam por sistemas de classificação após o beneficiamento, os grãos quebrados são separados, obtendo-se assim os subprodutos destes grãos, conhecidos popularmente como quirera de arroz e bandinha de feijão. Estes subprodutos têm pouca aceitação pelo consumidor, devido sua aparência e características físicas, apresentando baixo valor comercial, normalmente um terço do obtido pelo grão inteiro (NICOLETTI, 2007; LIMBERGER et al., 2008).

$\mathrm{O}$ aproveitamento industrial desses subprodutos como matéria-prima na obtenção de produtos alimentícios é uma alternativa viável, uma vez que apresentam qualidade nutricional semelhante à dos grãos inteiros. Assim, é extremamente importante o estímulo de pesquisas que contemplem o uso desses subprodutos como ingredientes a serem explorados pela indústria na alimentação humana (NICOLETTI, 2007).
Devido aos fatores antinutricionais que o feijão apresenta, torna-se necessário o emprego de tratamento térmico antes do consumo, geralmente cozimento. Esse tratamento térmico é empregado, tanto no âmbito doméstico como industrial, inativando substâncias tóxicas, aumentando a digestibilidade pela desnaturação das proteínas e gelatinização do amido. Alguns trabalhos têm sido realizados aplicando o processo de extrusão como tratamento térmico para o feijão. A extrusão promove a gelatinização do amido, o que resulta em grande absorção de água, permitindo também a proteólise mais extensiva e favorecendo a digestibilidade das proteínas (CARDOSO FILHO; CIACCO; SGARBIERI, 1993; CARDOSO FILHO; CIACCO; SGARBIERI, 1996).

A extrusão é um processo de alta temperatura e curto tempo de residência, podendo ocorrer, de acordo com as condições de processo utilizadas, reações como a de Maillard e menores perdas de nutrientes (CEREDA; VILPOUX; FRANCO, 2003; BOMBO, 2006). O processo de extrusão pode provocar diferentes reações, como destruição de aminoácidos por oxidação, principalmente dos que contêm enxofre, modificação de ligações entre aminoácidos, o que pode retardar sua liberação no processo digestivo, e formação de ligações nãohidrolisáveis durante a digestão. Esse processo também pode agir positivamente, possibilitando a inativação enzimática e destruição de fatores antinutricionais do feijão. A maior parte das substâncias tóxicas de leguminosas podem ser inativadas ou destruídas sob a ação do calor (GUALBERTO, 1981; LLO; SCHOENLECHNER; BERGHOFE, 2000).

O desafio da indústria de alimentação é desenvolver produtos de conveniência, como 
cereais matinais e snacks de alto valor nutricional, usando matérias-primas alternativas para atender às necessidades da população. Os salgadinhos extrusados incluem uma variedade de produtos com diferentes formas, que tiveram seu consumo aumentado devido à praticidade, sendo consumidos principalmente entre as refeições. A nova geração de salgadinhos extrusados tenta promover uma imagem de saúde com baixo teor de gordura e alto teor de fibras (CEREDA; VILPOUX; FRANCO, 2003). A produção de salgadinhos no Brasil está ganhando importância e o seu potencial de crescimento no mercado é promissor (LIMBERGER, 2006).

O objetivo desse trabalho foi estudar as características físicas, sensoriais e nutricionais de salgadinhos extrusados obtidos a partir de diferentes formulações de farinhas de quirera de arroz (FQA) e de bandinha de feijão (FBF), visando ampliar a utilização destes subprodutos na alimentação humana.

\section{Material e Métodos}

As farinhas usadas como matérias-primas foram obtidas a partir de quirera de arroz (Oryza sativa L. cv. BRS Primavera) e bandinha de feijão (Phaseolus vulgaris cv. Carioca).

A seleção dos subprodutos foi realizada manualmente, eliminando-se as sujidades e fragmentos mofados e escurecidos. Logo após, foram passadas separadamente por moinho de facas Versati, com peneira de diâmetro de 0,8 $\mathrm{mm}$. As farinhas obtidas foram acondicionadas, em embalagens laminadas compostas de filmes laminados de polietileno de baixa densidade e nylon (PEBD/ Nylon/ PEBD), e armazenadas à temperatura de $5 \pm 1{ }^{\circ} \mathrm{C}$, até o processamento.

Para formulação dos salgadinhos com farinha de quirera de arroz (FQA) e farinha de bandinha de feijão (FBF) utilizou-se delineamento inteiramente casualisado (DIC), com cinco tratamentos (0\% FQA/ 100\% FBF; 25\% FQA/ 75\% FBF; 50\% FQA/
$50 \%$ FBF; 75\% FQA/ 25\% FBF e 100\% FQA/ $0 \% \mathrm{FBF}$ ) e três repetições. Avaliaram-se o índice de expansão (dezesseis replicatas), a composição centesimal e o valor energético (triplicata).

Para elaboração dos salgadinhos extrusados, as formulações (bateladas de $1 \mathrm{~kg}$ ) foram condicionadas para $15 \%$ de umidade. O condicionamento foi realizado manualmente, colocando-se a água necessária com pulverizador, misturando-se durante $10 \mathrm{~min}$, com auxílio de sacos plásticos com volume de 4 L. Em seguida, as formulações foram embaladas em sacos laminados (PEBD/ Nylon/ PEBD) e armazenadas a temperatura de $5 \pm 1{ }^{\circ} \mathrm{C}$, até a elaboração dos salgadinhos extrusados.

A extrusão foi realizada na Empresa Micos Cicopal, situada em Senador Canedo, GO, em extrusora de rosca simples Inbramaq INBRA 140 (capacidade de produção de 110-140 $\mathrm{kg} / \mathrm{h}$ ), utilizando-se temperatura de $110 \pm 5{ }^{\circ} \mathrm{C}$ e diâmetro da matriz de $4 \mathrm{~mm}$. O processo foi efetuado em condições de processamento e de umidade constantes, permanecendo dessa forma variável somente a formulação do salgadinho. Após a extrusão, foi feita a secagem em estufa de convecção de ar forçada à temperatura de $60^{\circ} \mathrm{C}$, por 6 h. Cada amostra do produto, depois de resfriada, foi embalada em sacos laminados (PEBD/ Nylon/ PEBD) e armazenada à temperatura ambiente até a aromatização. Essa etapa foi realizada no dia anterior à análise sensorial. Para a aromatização dos salgadinhos foi empregada a formulação: $6 \%$ de óleo de canola, $4 \%$ de aroma de queijo (em pó), $1 \%$ de glutamato monossódico e $2 \%$ de sal refinado. Homogeneizou-se o óleo, o aroma e o glutamato monossódico em liquidificador doméstico ARNO Optimix, em bateladas de $300 \mathrm{~g}$, durante $5 \mathrm{~min}$. Em seguida, os salgadinhos foram pulverizados manualmente com essa mistura e adicionou-se o sal refinado. Os produtos obtidos (tratamentos) foram armazenados em sacos laminados (PEBD/ Nylon/ PEBD), até realização das análises.

Os tratamentos foram analisados quanto aos teores de umidade, de acordo com a técnica descrita 
pelo Instituto Adolf Lutz (1985); o nitrogênio total, segundo o método Kjeldahl, utilizando-se o fator 5,95 para arroz e 5,4 para feijão, para a conversão em proteína bruta (AOAC, 1990); o extrato etéreo, determinado por meio do método de Soxlet (AOAC, 1995); e o rendimento mineral fixo (cinzas), de acordo com método da AOAC (1990). Os carboidratos totais foram estimados por diferença, subtraindo-se de 100 os valores de proteínas, lipídeos e cinzas. O valor energético total dos alimentos processados foi estimado considerando-se os fatores de conversão de Atwater de $4 \mathrm{kcal} / \mathrm{g}$ de proteína, $4 \mathrm{kcal} / \mathrm{g}$ de carboidratos e $9 \mathrm{kcal} / \mathrm{g}$ de lipídeos (BRASIL, 2003). O índice de expansão radial foi avaliado no material $1 \mathrm{~h}$ após o processamento, sendo calculado pela relação entre o diâmetro médio da amostra e o diâmetro da matriz do extrusor, por meio de um paquímetro, em 16 salgadinhos separados aleatoriamente de cada parcela (VILELA; EL-DASH, 1987).

Os dados físicos e químicos obtidos foram avaliados por meio da análise de regressão, utilizando-se o aplicativo SAEG (2007). Os provadores analisaram os salgadinhos pelo teste de aceitabilidade, utilizando delineamento com blocos casualizados, sendo cada provador considerado um bloco. A avaliação dos salgadinhos ocorreu entre os dias 27 de agosto e 03 de setembro de 2008, na filial do Supermercado Marcos do Setor Serrinha em Goiânia-GO, utilizando 100 provadores para cada um dos cinco tratamentos, esses foram selecionados em função da disponibilidade e interesse. A análise foi realizada por qualquer pessoa que transitasse pelo local e demonstrasse interesse pelo produto. Não foram escolhidos provadores por sexo ou idade. $\mathrm{Na}$ avaliação, cada provador recebeu uma porção de apenas um salgadinho (tratamento), em um copo plástico descartável de $50 \mathrm{~mL}$, empregandose portanto, avaliação monádica. A ficha de análise sensorial continha, além do questionamento sobre a intenção de compra, questões relacionadas à aparência, aroma, textura e sabor para avaliação dos provadores. Utilizou-se escala hedônica estruturada em 9 pontos, sendo 1 (desgostei muitíssimo), 5 (indiferente) e 9 (gostei muitíssimo) (MEILGAAR; CIVILLE; CARR, 1999).

\section{Resultados e Discussão}

Os índices de expansão dos salgadinhos podem ser visualizado pelo gráfico de regressão (Figura 1). Houve aumento do índice de expansão com o aumento da quantidade de FQA e a redução da quantidade de FBF na composição dos salgadinhos.

$\mathrm{O}$ índice de expansão variou de entre 2,0 e 3,9. O tratamento $75 \% \mathrm{FQA} / 25 \% \mathrm{FBF}$ apresentou o maior índice de expansão, superior ao valor de 3,3 obtido por Asare et al. (2004) em snack com 70\% de farinha de arroz polido, $20 \%$ de farinha de feijão e $10 \%$ de amendoim. O tratamento 50\% FQA/ 50\% FBF obteve índice de expansão de 3,35 se assemelhando ao valor de 3,3, constatado por Boonyasirikool e Charunuch (2000) em snack de 50\% milho e 50\% quirera de arroz. Capriles, Soares e Arêas (2007) verificaram índice de expansão inferior ao valor de 6 em snacks com milho, valores acima dos obtidos neste trabalho.

De acordo com Karam, Grossmann e Silva (2001), o volume específico está diretamente relacionado com a expansão, que é a soma das expansões radial e axial. Logo, o grau de expansão é um fator importante a ser monitorado na produção de salgadinhos extrusados, principalmente devido a sua influência no peso e volume das embalagens. Índice de expansão e volume específico são relacionados com a gelatinização do amido, pois durante a passagem pelo equipamento ocorre a gelatinização e com a subseqüente descompressão na saída do extrusor ocorre a expansão (ALVES; GROSSMANN, 2002). A estrutura de produtos extrusados expandidos depende do grau de gelatinização do amido e da fusão deste no interior do extrusor, pois a gelatinização, seguida da fusão, aumentam as características elásticas da massa. Com isso, o vapor de água gerado durante o processo é responsável pela formação de bolhas no 
interior da massa fundida que são liberadas na saída da matriz, o que influencia diretamente a expansão dos produtos extrusados (FERREIRA, 2006).

$\mathrm{Na}$ figura 2 é apresentada a representação gráfica dos escores médios de aparência, aroma, textura e sabor dos salgadinhos elaborados a partir de FQA e ou FBF.

Em relação à aparência, textura e sabor os tratamentos 100\% FQA/ 0\% FBF, 75\% FQA/ 25\% FBF e 50\% FQA/ 50\% FBF foram superiores e não apresentaram diferença significativa entre si $(\mathrm{P}>0,05)$, obtendo para aparência escores entre 7-8 (gostei moderadamente - gostei muito) e para textura escores entre 8-9 (gostei muito - gostei muitíssimo), enquanto que para sabor o salgadinho elaborado somente com quirera de arroz obteve escore médio entre 8-9 e aqueles com 75\% FQA/ $25 \% \mathrm{FBF}$ e $50 \%$ FQA/ $50 \%$ FBF entre 7 e 8 . Em relação ao aroma, os tratamentos com 100\% FQA/ $0 \% \mathrm{FBF}$ e $75 \% \mathrm{FQA} / 25 \% \mathrm{FBF}$ foram superiores e também não diferiram entre si $(\mathrm{P}>0,05)$, obtendo escores médios entre 7-8.

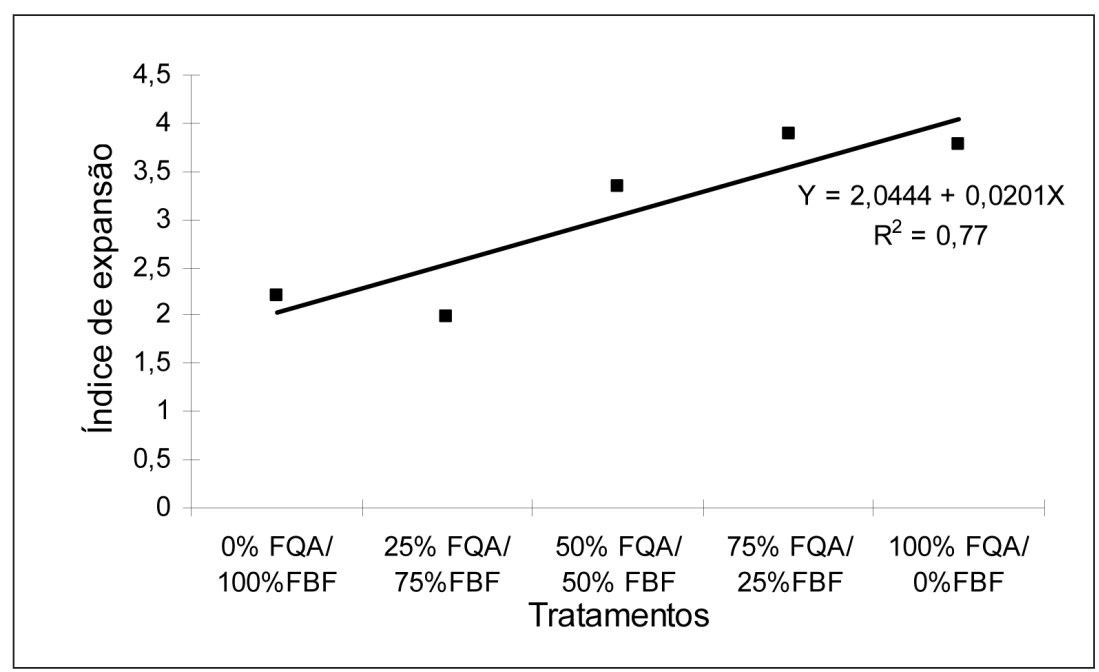

Figura 1. Índice de expansão dos salgadinhos em função da concentração de farinha de quirera de arroz (FQA).

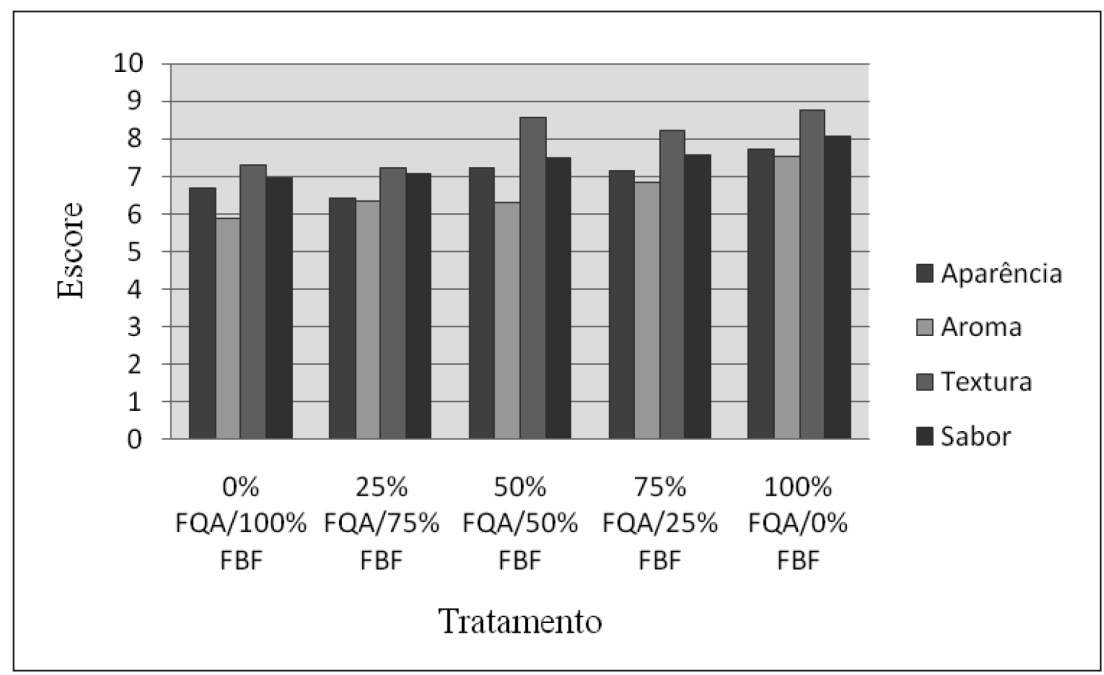

Figura 2. Escores médios de aparência, aroma, textura e sabor dos salgadinhos elaborados a partir de farinha de quirera de arroz (FQA) e ou farinha de bandinha de feijão (FBF). 
Os produtos com maior aceitabilidade em relação à aparência apresentaram em sua composição quantidade de FQA igual ou maior que FBF, o que os tornou mais claros e com maior índice de expansão (Figura 1), portanto, mais próximos das características dos salgadinhos obtidos a partir de grits de milho. A aparência é o primeiro atributo sensorial que o consumidor observa no momento da compra, sendo fundamental na escolha do produto, pois não é possível provar e avaliar os demais atributos. Assim, normalmente opta-se pelo produto com aparência e coloração mais atrativas (LIMBERGER, 2006).

O fato dos salgadinhos com 100\% FQA/ 0\% FBF e com $75 \%$ FQA $/ 25 \%$ FBF terem apresentado maior aceitabilidade em relação ao aroma provavelmente se explica pela predominância do amido em sua composição, que possui sabor e aroma neutro, enquanto aqueles com maiores teores de FBF apresentam teores de proteínas mais elevados. O teor de proteínas do feijão é bem maior que o do arroz, após a extrusão as proteínas sofrem ruptura, formando provavelmente compostos voláteis, o que favorece a percepção mais pronunciada de aroma “desagradável". O aromatizante de queijo utilizado não foi suficiente para mascarar totalmente esse aroma "desagradável", conforme as observações de alguns provadores.

Segundo Limberger (2006) e Tonelli (1995), a farinha de arroz, como material cru para extrusão, devido ao seu teor de amido oferece boa qualidade de expansão (Figura 1), possibilitando a obtenção de produtos extrusados com ótimas propriedades texturais. É notável e bem conhecida a grande diferença entre os produtos extrusados amiláceos e os protéicos. A homogeneidade estrutural do amido com apenas um tipo de monômero, capaz principalmente de interações do tipo eletrostáticas, faz com que se tenha uma série de condições favoráveis para a sua extrusão. Ao contrário das proteínas, amidos formam na extrusão uma massa fundida com características reológicas que garantem fluxo contínuo no equipamento, permitindo condições estáveis de operação (ARÊAS, 1996) e a obtenção de produtos mais homogêneos e de qualidade sensorial superior.

Os tratamentos com FBF em sua composição apresentaram gosto característico mais forte, que aumentou gradativamente com o aumento da quantidade deste subproduto. Os salgadinhos extrusados com 100\% FQA/ 0\% FBF, 75\% FQA/ $25 \% \mathrm{FBF}$ e $50 \%$ FQA/ $50 \%$ FBF apresentaram maior intenção de compra (80\%, 78\% e 76\%), enquanto os com 25\% FQA/ 75\% FBF e 0\% FQA/ $100 \%$ FBF tiveram maior rejeição, 39\% e 40\% dos provadores, respectivamente, não comprariam os produtos.

Na figura 3 observa-se que houve aumento de umidade e de carboidratos e diminuição de cinzas, de proteínas e de extrato etéreo dos salgadinhos, com a elevação da quantidade de FQA e consequente redução da quantidade de FBF na formulação dos mesmos. Os modelos matemáticos da variação de umidade, cinzas, proteínas, extrato etéreo e carboidratos em função do teor de FQA foram significativos, com coeficientes de determinação e de variação aceitáveis (Tabela 1).

Tabela 1. Modelos matemáticos, significância, coeficiente de determinação e de variação para umidade, cinzas, proteínas, lipídeos e carboidratos .

\begin{tabular}{llccc}
\hline & \multicolumn{1}{c}{ Modelo } & $\mathbf{P}$ & $\mathbf{R}^{\mathbf{2}}$ & $\mathbf{C V}$ \\
\hline Umidade & $\mathrm{Y}=4,4278+0,0708 \mathrm{X}$ & 0,0000 & 0,88 & 0,81 \\
Cinzas & $\mathrm{Y}=7,3654-0,038 \mathrm{X}$ & 0,0000 & 0,90 & 8,05 \\
Proteínas & $\mathrm{Y}=10,1742-0,0259 \mathrm{X}$ & 0,0000 & 0,88 & 2,37 \\
Extrato Etéreo & $\mathrm{Y}=5,6580-0,0211 \mathrm{X}$ & 0,0000 & 0,73 & 6,32 \\
Carboidratos & $\mathrm{Y}=72,3744+0,0779 \mathrm{X}$ & 0,0000 & 0,91 & 0,62 \\
\hline
\end{tabular}

$\mathrm{X}=$ teor de FQA $\left(\mathrm{g} 100 \mathrm{~g}^{-1}\right)$ 


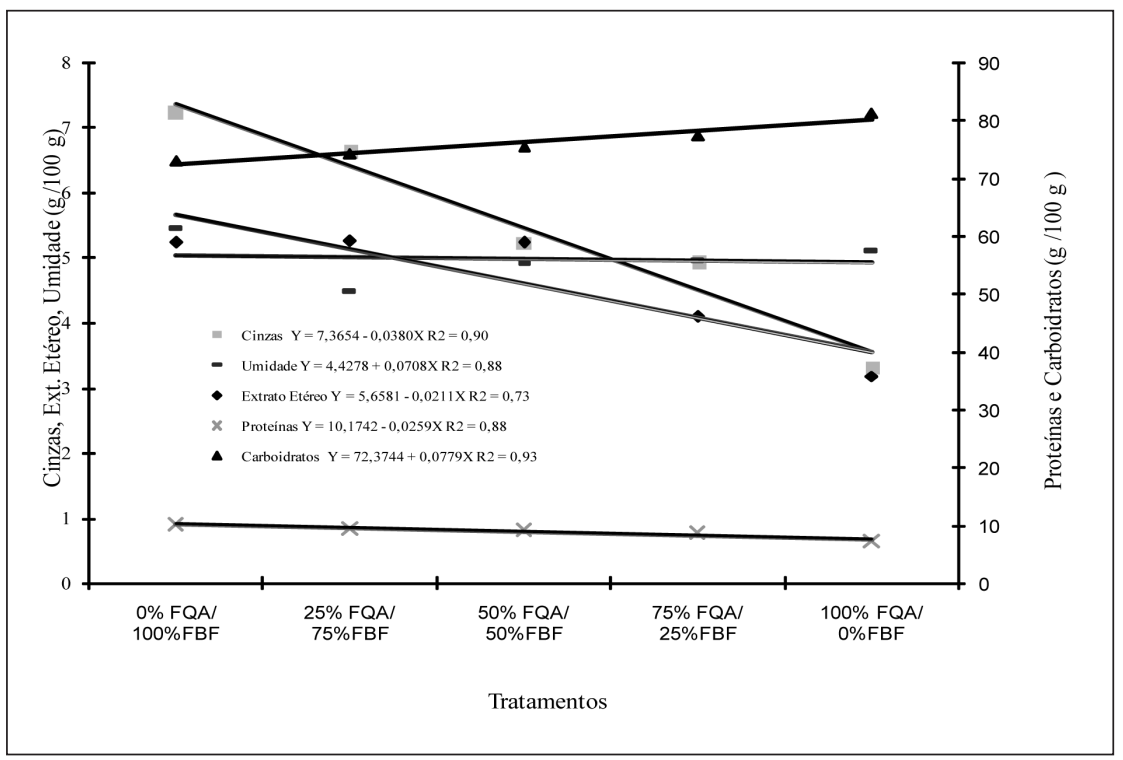

Figura 3. Teor de nutrientes em função do teor de farinha de quirera de arroz (FQA).

A umidade variou entre $4,5-5,1 \%$. A tendência de elevação do teor de umidade dos salgadinhos com o aumento da quantidade de FQA, devese provavelmente à maior umidade presente na farinha de quirera de arroz. De acordo com o teor encontrado por Ding et al. (2005), a farinha de arroz possui umidade de $12,3 \%$, sendo superior à umidade de farinha de feijão de $11,04 \%$ encontrada por Cardoso Filho, Ciacco e Sgarbieri (1996). O teor de umidade do tratamento $100 \% \mathrm{FQA} / 0 \% \mathrm{FBF}$ foi de $5,1 \%$ e está de acordo com o constatado por Limberger (2006).

As cinzas variaram entre $3,3-7,2 \%$. Com o aumento da quantidade de FQA, ocorre diminuição expressiva no teor de cinzas. Segundo Mesquita et al. (2007), o teor de cinzas no grão de feijão varia de $2,97 \%$ a $4,86 \%$, enquanto na farinha de arroz é de $0,78 \%$ de acordo com Ascheri, Ascheri e Carvalho (2006), o que explica os resultados obtidos neste trabalho.

As proteínas variaram entre $7,18 \%$ e $10,0 \%$. Houve elevação do teor proteínas nos salgadinhos em função do aumento da quantidade de FBF na formulação. Isso se deve ao elevado teor de proteínas presente no grão de feijão de 20,35\% a 27,34\%, observado por Toledo e Canniatti-Brazaca
(2008). Os tratamentos com FBF apresentaram teores de proteína mais elevados que o snack extrusado de milho, que contém, de acordo com Bombo (2006), cerca de 7,5\%. Os teores de proteína de todos os tratamentos foram superiores ao valor de 4,8\% encontrado por Ferreira (2006) em snack com $87,7 \%$ milho e $12,3 \%$ de farelo de trigo.

O extrato etéreo variou entre 3,2 e 5,3\% nos salgadinhos. Notou-se que, com o aumento da quantidade de FBF, houve elevação da quantidade de extrato etéreo (Figura 2). Isso se deve à maior quantidade de lipídeos presente na farinha de feijão, 2,1\%, conforme Cardoso Filho, Ciacco e Sgarbieri (1993), em relação ao teor de lipídeos na farinha de arroz, que varia de $0,3 \%$ a $0,5 \%$, segundo Llo, Schoenlechner e Berghofe (2000). A fabricação dos salgadinhos aumentou de forma significativa o teor de lipídeos quando comparado com os teores presentes nos grãos de arroz e feijão, devido à adição de óleo de canola para a saborização dos snacks. O salgadinho com $100 \%$ de FQA apresentou 3,2\% de lipídios, menor que o do salgadinho extrusado de quirera de arroz (8,1\%) obtido por Limberger (2006), provavelmente devido à diferença na quantidade de óleo adicionada na saborização dos produtos. Os tratamentos com $75 \%$ e $100 \%$ FQA, com 3,2\% e $4,1 \%$ de lipídios respectivamente, apresentaram 
teor de extrato etéreo inferior ao do snack de $50 \%$ de amaranto integral e 50\% de arroz polido (4,8\%), obtido por Ascheri, Carvalho e Spehar (2004).

Os carboidratos variaram entre 73 e $81 \%$, e aumentou com a elevação da quantidade de FQA e redução de FBF (Figura 2), devido ao arroz possuir maior quantidade de carboidratos em sua composição. A farinha de arroz polido apresenta $76 \%$ de carboidratos segundo Ascheri et al. (2006), sendo superior ao teor de carboidratos do feijão cru, de 51,7\%, encontrado por Cardoso Filho, Ciacco e Sgarbieri (1996). Todos os tratamentos obtiveram teor de carboidratos superior ao teor de 53\% do snack de milho, constatado por Bombo (2006).

A variação dos valores energéticos entre os tratamentos foi pequena, entre 379,5-385,6 $\mathrm{kcal} / 100 \mathrm{~g}$. De acordo com a análise de regressão, o modelo para o valor energético não foi significativo. $\mathrm{O}$ valor energético total de todos os tratamentos apresentaram-se acima dos valores energéticos de $358 \mathrm{kcal} / 100 \mathrm{~g}$ em snack light de milho (isento de lipídeos)e $305 \mathrm{kcal} / 100$ gdo snack com $70 \%$ de milho e 30\% de linhaça, determinados por Capriles, Soares e Arêas (2007) e Bombo (2006), respectivamente. Os valores energéticos totais obtidos neste trabalho podem estar superestimados, devido à presença de fibras, que não foram determinadas. Porém, os resultados foram inferiores a valores citados por Capriles, Soares e Arêas (2007), que variam entre

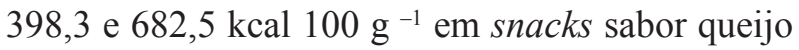
encontrados no mercado.

Pode-se concluir que é viável o aproveitamento dos fragmentos (subprodutos) de arroz e feijão como matéria-prima para desenvolver salgadinhos extrusados com características físicas, químicas e sensoriais adequadas para a alimentação humana, além de baixo custo, independente da proporção utilizada na formulação de farinhas de quirera de arroz e bandinha de feijão. Os salgadinhos extrusados foram aceitos pelos consumidores, sendo maior a aceitação quanto maior o teor de farinha de quirera de arroz na formulação. Com a adição de farinha de bandinha de feijão na composição dos salgadinhos houve aumento significativo do valor nutricional (cinzas, lipídios e proteínas) dos mesmos, sem elevação do valor energético.

\section{Agradecimentos}

À FAPEG pelo auxílio financeiro, à Capes pela bolsa de pós-doutorado e apoio financeiro, e à Empresa Micos Cicopal pela parceria.

\section{Referências}

ALVES, R. M. L.; GROSSMANN, M. V. E. Parâmetros de extrusão para produção de "snacks" de farinha de cará. Ciência e Tecnologia de Alimentos, Campinas, v. 22, n. 1, p. 32-38, 2002.

ARÊAS, J. A. G. Interações moleculares do amido durante o processo de extrusão. Boletim da Sociedade Brasileira de Ciência e Tecnologia de Alimentos, Campinas, v. 30, n. 1, p. 28-30, 1996.

ASARE, E. K.; SEFA-DEDEH, S.; SAKYI-DAWSON, E.; AFOAKWA, E. O. Application of response surface methodology for studying the product characteristics of extruded rice-cowpea-groundnut blends. Journal of Food Sciences and Nutrition, Chicago, v. 55, n. 5, p. 431-439, 2004.

ASCHERI, D. P. E.; ANDRADE, C. T.; CARVALHO, C. W. P.; ASCHERI, J. L. R. Efeito da extrusão sobre a adsorção de água de farinhas mistas pré-gelatinizadas de arroz e bagaço de jabuticaba. Ciência e Tecnologia de Alimentos, Campinas, v. 26, n. 2, p. 325-335, 2006.

ASCHERI, D. P. R; ASCHERI, J. L. R; CARVALHO, C. W. P. de. Caracterização da farinha de bagaço de jabuticaba e propriedades funcionais dos extrusados. Ciência e Tecnologia de Alimentos, Campinas, v. 26, n. 4, p. 897-905, 2006.

ASCHERI, J. L. R.; CARVALHO, C. W. P. de; SPEHAR, C. R. A extrusão do amaranto no desenvolvimento de produtos: caracterização físico-química. Rio de Janeiro: Embrapa Agroindústria de Alimentos, 2004. 32 p. (Documentos, 60).

ASSOCIATION OF OFFICIAL ANALYTICAL CHEMISTS. AOAC. Oficial methods of analyses of the Association of Official Analytical Chemists. 15. ed. Arlington: AOAC, 1990. 
Oficial methods of analyses of the Association of Official Analytical Chemists. 16. ed. Arlington: AOAC, 1995.

BOMBO, A. J. Obtenção e caracterização nutricional de snacks de milho (Zea mays L.) e linhaça (Linum usitatissimum L.). 2006. Dissertação (Mestrado em Saúde Pública) - Universidade de São Paulo, São Paulo.

BOONYASIRIKOOL, P.; CHARUNUCH, C. Development of corngrit-broken Rice based snack food by extrusion cooking. Kasetsart Journal: Natural Sciences, Bangkok, v. 34, p. 279-288, 2000.

BRASIL. Agência Nacional de Vigilância Sanitária. Resolução RDC n. 360, de 23 de dezembro de 2003. Aprova regulamento técnico sobre rotulagem nutricional de alimentos embalados, tornando obrigatória a rotulagem nutricional. Disponível em: <http://e-legis.anvisa.gov.br/ leisref/public/show.Act.php?id=9059>. Acesso em: 18 nov. 2003.

CAPRILES, V. D.; SOARES, R. A. M.; ARÊAS, J. A. G. Development and assessment of acceptability and nutritional properties of a light snack. Ciência $e$ Tecnologia de Alimentos, Campinas, v. 27, n. 3, p. 562566, 2007.

CARDOSO FILHO, N.; CIACCO, C. F.; SGARBIERI, V. C. Efeito das variáveis do processo de extrusão nas características nutricionais das proteínas do feijão comum. Ciência e Tecnologia de Alimentos, Campinas, v. 13, n. 2, p. 142-150, 1993.

Efeito de algumas variáveis do processo de extrusão nas características tecnológicas da farinha do feijão comum. Ciência e Tecnologia de Alimentos, Campinas, v. 16, n. 2, p. 158-164, 1996.

CEREDA, M. P.; VILPOUX. O.; FRANCO, C. M. L. Uso de amido e seus derivados na produção de salgadinhos extrusados (snacks). In: CEREDA, M. P.; VILPOUX, O. F. (Org.). Tecnologia, usos e potencialidades de tuberosas amiláceas Latino Americanas. São Paulo: Fundação Cargill, 2003, v. 3, p. 132-142.

DING, Q.; AINSWORTH, P.; TUCKER, G.; MARSON, H. The effect of extrusion conditions on the physicochemical properties and sensory characteristics of rice-based expanded snacks. Journal of Food Engineering, Oxford, v. 66, n. 3, p. 283-289, 2005.

FERREIRA, R. E. Avaliação de parâmetros do processo de extrusão e do teor de farelo de trigo adicionado em características de 'snacks' expandidos de milho. 2006. Dissertação (Mestrado em Tecnologia de Alimentos) Universidade Estadual de Campinas, Campinas.
FOOD AND AGRICULTURAL ORGANIZATION FAO. Rice around the world: Brazil, 2004. Disponível em: <http://www.fao.org/RICE2004/en/p1.htm>. Acesso em: 20 jun. 2008.

GUALBERTO, D. G. Avaliação nutricional e sensorial de misturas de feijão e soja processados por extrusão. 1981. Dissertação (Mestrado em Ciência e Tecnologia de Alimentos) - Universidade Federal de Viçosa, Viçosa, MG.

INSTITUTO ADOLFO LUTZ. Normas analiticas do Instituto Adolfo Lutz: métodos químicos e físicos para análise de alimentos. 2. ed. São Paulo: IMESP, 1985. 533 p.

KARAM, L. B.; GROSSMANN, M. V. E.; SILVA, R. S. S. F. Misturas de farinha de aveia e amido de milho com alto teor de amilopectina para produção de "snacks". Ciência e Tecnologia de Alimentos, Campinas, 2001, v. 21, n. 2, p. 158-163, 2001.

LAJOLO, F. M.; GENOVESE, M. I.; MENEZES, E. W. de. Qualidade nutricional. In: ARAUJO, R. S.; RAVA, C. A.; STONE, L. F.; ZIMMERMANN, M. J. O. de. (Coord.).Cultura do feijoeiro comum no Brasil. Piracicaba: POTAFÓS, 1996. p. 24-56.

LIMBERGER, M. V. Modificação física e química do amido de quirera de arroz para aproveitamento na indústria de alimentos. 2006. Dissertação (Mestrado em Ciência e Tecnologia de Alimentos) - Universidade Federal de Santa Maria, MS.

LIMBERGER, V. M.; SILVA, L. P. da.; EMANUELLI, T.; COMARELA, C. G.; DALPIEVE, L. Modificação química e física do amido de quirera de arroz para aproveitamento na indústria de alimentos. Química Nova, São Paulo, v. 31, n. 1, p. 84-88, 2008.

LLO, S.; SCHOENLECHNER, R.; BERGHOFE, E. Role of lipids in the extrusion cooking processes. Grasas y Aceites, España, v. 51, n. 1/2, p. 97-110, 2000.

MEILGAAR, M.; CIVILLE, G. V.; CARR, B. T. Sensory evaluation techniques. 3. ed. Boca Raton: CRC Press, Inc., 1999, 387 p.

MESQUiTA, F. R.; CORRÊA, A. D.; ABREU, C. M. P. de.; LIMA, R. A. Z.; ABREU, A. de. F. B. Linhagens de feijão (Phaseolus vulgaris L.): composição química e digestibilidade protéica. Ciência e Agrotecnologia, Lavras, v. 31, n. 4, p. 1114-1121, 2007.

NICOLETTI, A. M. Enriquecimento nutricional de macarrão com uso de subprodutos agroindustriais de baixo custo. 2007. Dissertação (Mestrado em Ciência e Tecnologia de Alimentos) - Universidade Federal de Santa Maria. Santa Maria, RS. 
SAEG. Sistema para análises estatísticas e genéticas. Versão 9.1. Viçosa: Fundação Arthur Bernardes. 2007.

SILVA, C. C. da; DEL PELOSO, M. J. (Ed.). Informações técnicas para o cultivo do feijoeiro comum na região central-brasileira 2005-2007. Santo Antônio de Goiás: Embrapa Arroz e Feijão, 2006. 140 p. (Documentos, 193).

TOLEDO, T. C. F. de.; CANNIATTI-BRAZACA, S. G. Avaliação química e nutricional do feijão carioca (Phaseolus vulgaris L.) cozido por diferentes métodos. Ciência e Tecnologia de Alimentos, Campinas, v. 28, n. 2, p. 355-360, 2008.
TONELLI, E. G. A. de. Desenvolvimento de um equipamento e otimização do processo de produção de arroz expandido. 1995. Dissertação (Mestrado em Ciência dos Alimentos) - Universidade Federal de Lavras, Lavras, MG.

VILELA, E. R.; EL-DASH, A. A. Extrusão de farinha de guandu (Cajanus cojan, Millsp). Efeitos das variáveis do processo nas características químicas, físicas e fisicoquímicas dos produtos extusados. Revista da Sociedade Brasileira de Ciência e Tecnologia de Alimentos, Campinas, v. 7, n. 2, p. 97-116, 1987. 